\title{
Bridging the gap between modelling and control of anesthesia: an ambitious ideal
}

\author{
Clara M. Ionescu ${ }^{1}$, Dana Copot $^{1}$, Cosmin Copot $^{1}$ and Robin De Keyser ${ }^{1}$
}

\begin{abstract}
This work focuses on the problematic of modeling the drug diffusion process that occurs in the human body when an anesthetic drug is taken up. This is performed here using emerging tools from fractional calculus, i.e. by using fractionalorder impedance models (FOIMs). A novel interpretation is given to the classical pharmacokinetic and pharmacodynamic models for drug uptake in the body. The major challenges that are encountered during this development consist of online identification of the patient model, introducing a logarithmic sampling time and embedding the proposed method in a closed loop control system.
\end{abstract}

Index Terms-anesthesia, closed loop control, drug dosing, pharmacokinetic models, pharmacodynamic models, fractional order derivatives, fractional-order impedance model (FOIM)

\section{INTRODUCTION}

The last few decades, modern medicine has successfully been influenced by advanced control technologies resulting in applications such as robotic surgery, electro-physiological system life support and image-guided therapy and surgery. An interesting application of control in medicine is clinical pharmacology and in particular the control of general anesthesia [1]. Monitoring and controlling the depth of anesthesia for surgical patients poses interesting challenges to the control engineer [2] as it is a multi-variable interaction process that has long captured the attention of engineers and clinicians [3]. The first designs were expert systems that advised the anesthesiologist upon optimal drug infusion rates during clinical trials [4]. Control of anesthesia has a many challenges with multi-variable characteristics, different dynamics depending on anesthetic substances and stability problems [5]. The advantage of automated closed loop control of anesthesia is that it gives a continuous drug delivery, contrary to intermittent control which is nowadays standard practice. A continuous drug delivery ensures that there is no under- or over-dose of hypnotic or analgesic drugs that could result in patients that feel pain during surgery but are unable to react [6].

The research presented in this paper merges classical control theory with the young promising field of fractional-order modeling to measure pain relief levels in an unconscious patient and initiate the development of a biosensor for analgesia levels. A few pioneering attempts to measure the analgesic component of general anesthesia have shown that current

\footnotetext{
${ }^{1}$ The authors are with the Department of Electrical Energy, Systems and Automation, Ghent University, Technologiepark 913, 9052 Gent, Belgium, e-mail: \{Cosmin.Copot, Dana.Copot, ClaraMihaela.Ionescu, Robain.DeKeyser\}@UGent.be
}

state-of-art is unable to deliver suitable signals and models for optimal regulation [7]. The result is then a high risk of drug over- or under-dosing and unwanted post-operatively effects, leading to increased hospitalization and health-care costs for both society and patient [8]. The pharmacokinetic (PK) models available in the literature for anesthesia are linear in terms of model parameters and dynamics [9], [10]. Their frequency response is quasi-identical, less for a scaling factor in the gain (i.e. this accounts in part for the sensitivity to the drug with respect to the body mass index of the patient). The pharmacodynamic (PD) models, are usually represented by nonlinear Sigmoid (Hill) curves and represent the relationship of the drug concentration to the drug effect in each patient [7]. From patient-individualised control point of view, PD models are the most challenging part of the patient model and pose most challenges for control (i.e. highly nonlinear characteristic).

Fractional calculus offers tools to model such nonlinear characteristics as those of the PK-PD models with a smaller degree of nonlinearity in model parameters [11]. The overall purpose of this paper is to show a methodology which enables a different, fresh view to these models and demonstrates how they can be integrated in the control paradigm of depth of anesthesia regulation.

The paper is structured as follows: in section II, we describe analgesia and the coinciding diffusion process. Section III discusses the control method that will be used in combination with the proposed analgesia sensor and the possible models used in this control. The grand challenges in the development of the sensor are discussed in section IV and the conclusions are summarized in a final section.

\section{AnAlgesia: the Missing PIECE}

General or complete anesthesia refers to inhibition of sensory, motor and sympathetic nerve transmission at the level of the brain, resulting in unconsciousness and lack of sensation [5], [6], [12]. It consists of three components: hypnosis, analgesia and neuromuscular blockade. A schematic representation of the anesthesia paradigm in terms of its components is given in figure 1. The hypnosis [5], [13], [14] and neuromuscular blockade [15], [16] are wellcharacterized in terms of models, measured variables and closed loop control. The third component, i.e. analgesia, remains challenging for control purposes because no direct output measure is available [17]. Hence, no specific models 
are yet available for analgesia effect in the human body during general anesthesia (i.e. unconsciousness).

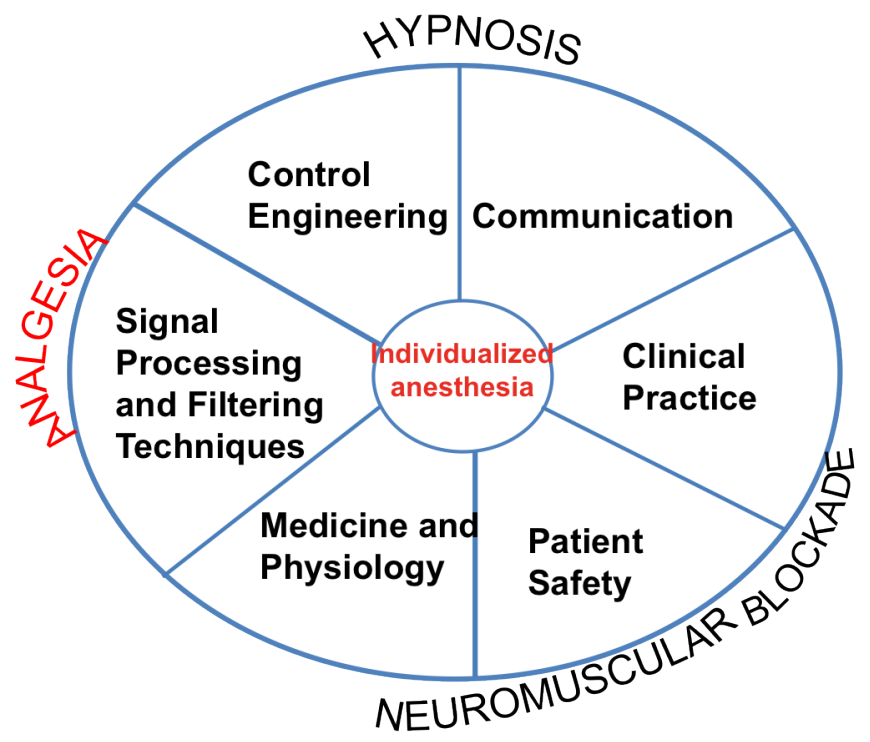

Fig. 1. A personal view of the anesthesia paradigm. The items in red are the current challenges.

Hypnosis is a general term indicating unconsciousness and absence of post operative recall of events occurred during surgery. The level of hypnosis is related with the infusion of hypnotic drugs such as propofol and can be monitored by BIS monitoring. BIS is a short-hand name for Bispectral Index, which is a measure of the electrical activity in the brain measured at the frontal and parietal bones of the skull by a sensor transducer plasture on the skin [5]. Analgesia is defined as an insensibility to pain without loss of consciousness. It is a state in which painful stimuli are not perceived or interpreted as pain and is usually induced by a drug. Neuromuscular blockade is induced to prevent unwanted movement or muscle tone and causes paralysis during surgical procedures. The muscle relaxants are given intravenously (through the bloodstream) and act directly on the muscles.

Hence, analgesia is the amount of pain relief achieved during general anesthesia. The pain relief is obtained by administrating an analgesic drug such as remifentanil to the patient. The effectiveness of the analgesic drugs relies on how they are able to block the neural messages to the brain that are sent by the pain receptors. At this moment there are no mathematical model to suggest an output effect of analgesic drugs. In this paper, a hypothetical model is discussed within the larger scope of closed loop control systems and integration of such models within these loops.

\section{Classical PK-PD MODELS}

In order to control the depth of anesthesia, and implicitly analgesia, by means of model based control strategies, a model which captures the dynamics of the patient is required. The selection of the model input and output variables is crucial for optimal control [14]. Propofol is a commonly used hypnotic drug to induce general anesthesia [6]. Remifentanil is an opioid with a unique pharmacologic profile, best characterized by its high metabolic clearance, independent of the most common metabolic pathways which are usually known to metabolize anesthetic drugs [18]. When administered together, these two drugs interact synergistically on both hypnotic and analgesic components of sedation. Using the electronencephalogram (EEG), several derived, computerised parameters like the BIS have been tested and validated as a promising measure of the hypnotic component of anesthesia [5], [19]. BIS values lie in the range of $0 \%-100 \%$; whereas $90 \%-100 \%$ range represents fully awake patients; $60 \%$ $70 \%$ range and $40 \%-60 \%$ range indicate light and moderate hypnotic state, respectively.

Pharmacokinetic (PK) and pharmacodynamic (PD) blocks denote compartmental models [20]. The PK-PD models most commonly used for propofol and remifentanil are the 4th order compartmental model described in [9], [10], [18] and they have the structure depicted in Fig. 2.

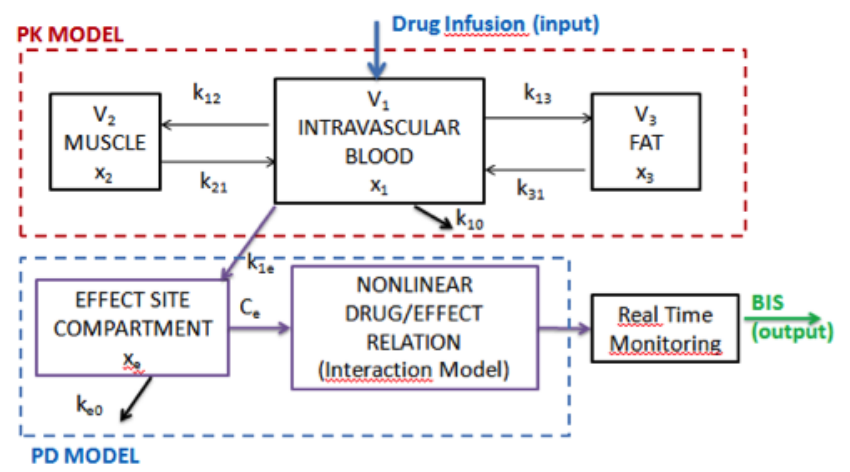

Fig. 2. General compartmental model of the patient, where PK denotes the pharmacokinetic model and PD denotes the pharmacodynamic model of an infused drug [22].

In this figure $x_{1}[\mathrm{mg}]$ denotes the amount of drug in the central compartment. The blood concentration is expressed by $x_{1} / V_{1}$. The peripheral compartments 2 and 3 model the drug exchange of the blood with well and poorly diffused body tissues. The masses of drug in fast and slow equilibrating peripheral compartments are denoted by $x_{2}$ and $x_{3}$, respectively. The parameters $k_{j i}$, for $i \neq j$, denote the drug transfer frequency from the $j^{\text {th }}$ to the $i^{\text {th }}$ compartment and $u(t)[\mathrm{mg} / \mathrm{s}]$ is the infusion rate of the anesthetic drug into the central compartment. An additional hypothetical effect compartment was proposed to represent the lag between drug plasma concentration and drug response. The concentration of drug in this compartment is represented by $x_{e}$. The parameters $k_{i j}$ of the PK models depend on age, weight, height and gender and the relations for Propofol can be found in [9], [10], respectively for Remifentanil in [18].

The PK-PD model is represented by the following equations [12], [20], [21]: 


$$
\begin{aligned}
\dot{x}_{1}(t)= & -\left(k_{10}+k_{12}+k_{13}\right) \cdot x_{1}(t)+k_{21} \cdot x_{2}(t)+ \\
& +k_{31} \cdot x_{3}(t)+u(t) / V_{1} \\
\dot{x}_{2}(t)= & k_{12} \cdot x_{1}(t)-k_{21} \cdot x_{2}(t) \\
\dot{x}_{3}(t)= & k_{13} \cdot x_{1}(t)-k_{31} \cdot x_{3}(t) \\
\dot{x}_{e}(t)= & -k_{e 0} \cdot x_{e}(t)+k_{1 e} \cdot x_{1}(t)
\end{aligned}
$$

The effect compartment receives drug from the central compartment by a first-order process and it is regarded as a volumeless additional compartment. Therefore, the drug transfer frequency from the central compartment to the effect-site compartment is equal to the frequency of drug removal from the effect-site compartment: $k_{e 0}=k 1 e=$ $0.456\left[\mathrm{~min}^{-1}\right]$ [5], [19]. The corresponding concentration of $x_{e}$ in the effect site compartment is denoted by $C_{e}$.

The parameters of the PK models depend on age, weight, height and gender and can be calculated for Propofol [9], [10]:

$$
\begin{aligned}
& V_{1}=4.27[l] \quad V_{3}=2.38[l] \\
& V_{2}=18.9-0.391 \cdot(\text { age }-53)[l] \\
& C_{l 1}=1.89+0.0456(\text { weight }-77)-0.0681(\text { lbm }-59)+ \\
& +0.0264(\text { height }-177)[l / m i n] \\
& C_{l 2}=1.29-0.024(\text { age }-53)[l / m i n] \\
& C_{l 3}=0.836[l / m i n] \\
& k_{10}=\frac{C_{l 1}}{V_{1}}\left[\mathrm{~min}^{-1}\right] ; k_{12}=\frac{C_{l 2}}{V_{1}}\left[\mathrm{~min}^{-1}\right] ; k_{13}=\frac{C_{l 3}}{V_{1}}\left[\mathrm{~min}^{-1}\right] \\
& k_{21}=\frac{C_{l 2}}{V_{2}}\left[\mathrm{~min}^{-1}\right] ; k_{31}=\frac{C_{l 3}}{V_{3}}\left[\mathrm{~min}^{-1}\right] \\
& k_{e 0}=0.456\left[\mathrm{~min}^{-1}\right]
\end{aligned}
$$

where $C_{l 1}$ is the rate at which the drug is cleared from the body, and $C_{l 2}$ and $C_{l 3}$ are the rates at which the drug is removed from the central compartment to the other two compartments by distribution. Similarly, for Remifentanil [18]:

$$
\begin{gathered}
V_{1}=5.1-0.0201(\text { age }-40)+0.072(\mathrm{lbm}-55)[l] \\
V_{2}=9.82-0.0811(\text { age }-40)+0.108(\mathrm{lbm}-55)[l] \\
V_{3}=5.42[l] \\
C_{l 1}=2.6+0.0162(\text { weight }-40)-0.0191(\mathrm{lbm}-55)[\mathrm{l} / \mathrm{min}] \\
C_{l 2}=2.05-0.0301(\text { age }-40)[\mathrm{l} / \mathrm{min}] \\
C_{l 3}=0.076-0.00113(\text { age }-40)[\mathrm{l} / \mathrm{min}] \\
k_{10}=\frac{C_{l 1}}{V_{1}}\left[\mathrm{~min}^{-1}\right] ; k_{12}=\frac{C_{l 2}}{V_{1}}\left[\mathrm{~min}^{-1}\right] ; k_{13}=\frac{C_{l 3}}{V_{1}}\left[\mathrm{~min}^{-1}\right] \\
k_{21}=\frac{C_{l 2}}{V_{2}}\left[\mathrm{~min}^{-1}\right] ; k_{31}=\frac{C_{l 3}}{V_{3}}\left[\mathrm{~min}^{-1}\right] \\
k_{e 0}=0.595-0.007(\text { age }-40)\left[\mathrm{min}^{-1}\right]
\end{gathered}
$$

The lean body mass (lbm) for men and for women have the following expressions:

$$
\begin{aligned}
& l b m_{m}=1.1 \cdot \text { weight }-128 \cdot \frac{\text { weight }}{h^{2}} \\
& l b m_{f}=1.07 \cdot \text { weight } \\
& l \text { weight } \\
& \text { height }
\end{aligned}
$$

respectively.

The BIS variable can be related to the drug effect concentration $C_{e}$ by the empirical static but time varying nonlinear relationship, called also the Hill curve [7]:

$$
B I S(t)=E_{0}-E_{\max } \cdot \frac{C_{e}(t)^{\gamma}}{C_{e}(t)^{\gamma}+C_{50}^{\gamma}}
$$

where $E_{0}$ denotes the baseline (awake state - without drug) value, which, by convention, is typically assigned a value of $100, E_{\max }$ denotes the maximum effect achieved by the drug infusion, $C_{50}$ is the drug concentration at half maximal effect and represents the patient sensitivity to the drug, and $\gamma$ determines the steepness of the curve. Some typical parameters extracted from medical expertise can be found in [5], [6], [22].

As explained earlier, the main challenge for control standpoint is the nonlinearity of the Hill curve given by (5) and the inherent inter-patient variability. To illustrate this to the reader, a realistic set of typical and atypical patients has been used, with the parameter values given in [22], as extracted from medical expertise. The resulted Hill curves by simulating the PKPD model of these patients is shown in figure 3 .

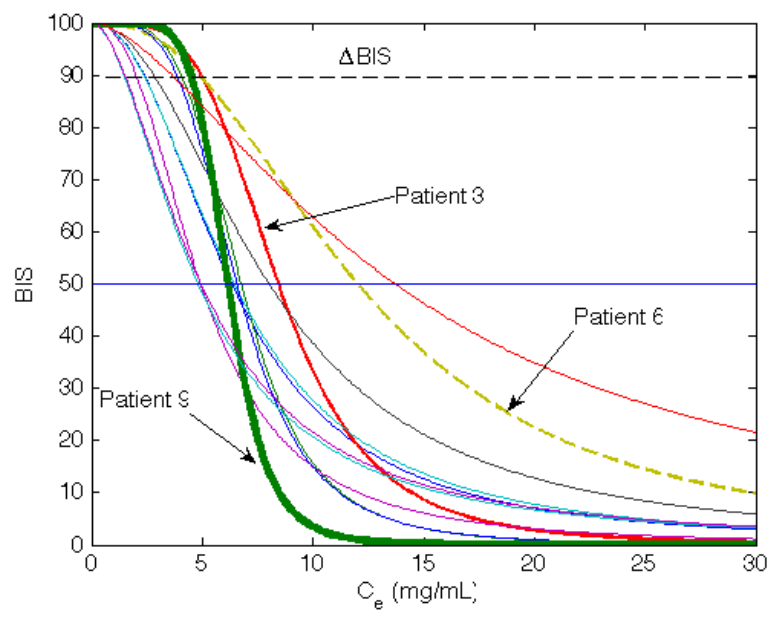

Fig. 3. Example of Hill curves from various patients.

\section{EFFECT TO DRUG CONCENTRATION RELATION}

] Pure empirical models such as input - output models are rather descriptive with loss of physiological insight into the drug absorption, transport, diffusion and release. However, from the control point of view they are the easiest to obtain and to deliver information to the controller for deciding the optimal drug infusion rate necessary for the specific patient whose model is available. On the other hand, physiologically and bio-chemically based models are difficult to attain due to the extraordinary complexity of this process. An intermediate solution is perhaps the use of semi-empirical models [23]. Such a model which is fairly simple to employ in characterizing drug release is the power-law model:

$$
\frac{M_{t}}{M_{\infty}}=k t^{n}
$$

where $M_{t}$ and $M_{\infty}$ are cumulative amounts of drug released at time $t$ and infinite time, respectively; and $k$ is a constant reflecting the structural and geometrical characteristics of the system, and $n$ is the so-called "release exponent" [24]. For 
purely diffusion-controlled release, $n=0.5$. other values of $n$ may be indicative of various diffusion conditions. These features of this model are of special interest in our study since we would like to look at the inter-patient variability from this standpoint.

Following the fundamental law of physics which applies to well stirred, homogeneous systems, it follows that the mean square displacement of the walker, $\left\langle x^{2}\right\rangle$ in the random walk model is proportional to time [23], [25]:

$$
<x^{2}>\propto t
$$

However, in disordered systems, such as most biological environments, this is no longer proportional with time, but with the fractal walk dimension of the walker:

$$
<x^{2}>\propto t^{D_{w}}
$$

with $D_{w} \neq 2$. This property implies that scaling laws such as power laws are associated with kinetics of various processes taking place in the (biological) environment (i.e. tissue). Most biological tissues can be approximated in their properties by polymers. As such, the spatio-temporal porosity of a dynamically changing polymer is close to the percolation threshold for non-classical diffusion effects impinging on release kinetics.

Fractional calculus has shown in several (non)biological applications that classical diffusion as well as non-classical diffusion can be characterized by fractional order differintegrals models [26]. It is possible to derive further the model from (6) in its Laplace equivalent, as indicated in [27]. This result has regained the attention of the research community and current efforts are being directed towards providing pharmacokinetic models with fractional order differ-integrals [11], [28], [29], [30], [31], [32], [33], and their equivalent Laplace models of non-integer order, coined in the literature as FOIMs (fractional order impedance models) [34].

\section{Proposed View on Patient Models}

\section{A. Emerging PK models}

Figure 4 presents a similar scheme as that from figure 2 for the PK model introduced in this section.

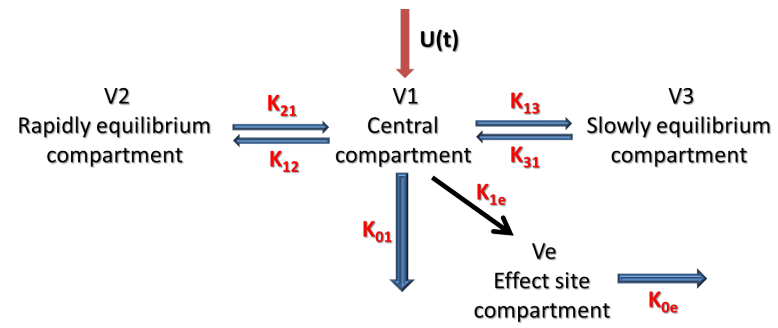

Fig. 4. Three-compartment model representing the basic pharmacokinetic processes that occur after intravenous drug administration. $U$, dosing scheme as a function of time; $k_{01}$, rate constant reflecting all processes acting to irreversibly remove drug from the central compartment; $K_{12}, K_{13}$, $K_{21}, K_{31}$ and $K_{1 e}$ represents the intercompartmental rate constants; $V 1$, represents the volume of the central compartment, $V 2$ and $V 3$ represents the volume of the peripheral compartments (muscle and respectively fat).
The pharmacokinetic model used in practice for drug concentration prediction is citedoko,doko1,popovic:

$$
\begin{gathered}
\dot{q}_{1}(t)=K_{21} q_{2}(t)+K_{31} q_{3}(t)-K_{12} q_{1}(t) \\
-K_{13} q_{1}(t)-K_{01} q_{1}(t)-K_{1 e} q_{1}(t)+U(t) \\
\dot{q}_{2}(t)=K_{12} q_{1}(t)-K_{21} q_{2}(t)-K_{02} q_{2}(t) \\
\dot{q}_{3}(t)=K_{13} q_{1}(t)-K_{31} q_{3}(t)-K_{03} q_{3}(t) \\
\dot{q}_{e}(t)=K_{1 e} q_{1}(t)-K_{0 e} q_{e}(t)
\end{gathered}
$$

where: $q_{1}, q_{2}$ and $q_{3}$ [mg] denotes the amount of drug in the three compartments and $V 1, V 2$ and $V 3$ represents the volume in the three compartments. The drug concentration in the blood is expressed by $q_{1} / V 1$, respectively $q_{2} / V 2$ and $q_{3} / V 3$ for compartment 2 and 3 . The peripheral compartments 2 (muscle) and 3 (fat) models the drug exchange of the blood with well and poorly diffused body tissues. The parameters $K_{i j}$ for $i j$, denote the drug transfer frequency from the i-th to the $j-t h$ compartment and $U(t)[\mathrm{mg} / \mathrm{s}]$ is the infusion rate of the analgesic drug into the first compartment. The parameters $K_{i j}$ of the PK models depend on age, weight, height and gender and can be calculated for Propofol as in [32], [33].

Standard arguments imply that the equations which are determined by the mass balance in compartments have the following form [29], [30].

$$
\begin{aligned}
& \tau_{1}^{n_{1}-1} D^{n_{1}} q_{1}(t)=K_{21} q_{2}(t)+K_{31} q_{3}(t) \\
& -K_{12} q_{1}(t)-K_{13} q_{1}(t)-K_{1 e} q_{1}(t)-K_{01} q_{1}(t)+U(t) \\
& \tau_{2}^{n_{2}-1} D^{n_{2}} q_{2}(t)=K_{12} q_{1}(t)-K_{21} q_{2}(t)-K_{02} q_{2}(t) \\
& \tau_{3}^{n_{3}-1} D^{n_{3}} q_{3}(t)=K_{13} q_{1}(t)-K_{31} q_{3}(t)-K_{03} q_{3}(t) \\
& \tau_{e}^{n_{e}-1} D^{n_{e}} q_{e}(t)=K_{1 e} q_{1}(t)-K_{0 e} q_{e}(t)
\end{aligned}
$$

Dividing equations (13)-(16) by $\tau_{i}^{n_{i}}$ and redefining the constants we obtain the following fractional order differential equations.

$$
\begin{gathered}
D^{n_{1}} q_{1}(t)=k_{21} q_{2}(t)+k_{31} q_{3}(t)-k_{12} q_{1}(t)- \\
-k_{13} q_{1}(t)-k_{1 e} q_{1}(t)-k_{01} q_{1}(t)+U(t) \\
D^{n_{2}} q_{2}(t)=k_{12} q_{1}(t)-k_{21} q_{2}(t)-k_{02} q_{2}(t) \\
D^{n_{3}} q_{3}(t)=k_{13} q_{1}(t)-k_{31} q_{3}(t)-k_{03} q_{3}(t) \\
D^{n_{e}} q_{e}(t)=k_{1 e} q_{1}(t)-k_{0 e} q_{e}(t)
\end{gathered}
$$

where: $\tau_{i}$ represents the characteristic time of the $i-t h$ compartment, expressed in seconds. $i=1, \ldots, n$ and $K_{i j}$, for $i, j=1, \ldots, n$ are standard diffusion coefficients having the dimension $1 / \mathrm{s}, n_{1}=n_{2}=n_{3}=n$ and the constants were redefined according to relation (21). The introduction of $\tau_{i}$ leads to the dimensional homogeneity of fractional rate equations.

$$
k_{i j}=\frac{K_{i j}}{\tau_{i}^{n_{1}-1}}
$$


Where $k_{i j}$ are the constants depending on the rate of absorption, elimination, tissue take up, characteristic and inactivation of drug. One of the characteristic of fractional derivatives is the "memory effect" term. It is well known that the state of many systems (biological, electrochemical, viscoelastic, etc.) at a given time depends of their configuration at previous time [29], [31].

This model has been successfully validated in simulations with values resulted from medical expertise against the classical model from (1) in [32], [33].

\section{B. Emerging PD models}

Based on (6) one can write the same relationship for the Hill curve [7], [35], [36]:

$$
\frac{C e(t)}{B I S(t)}=k \cdot t^{n}
$$

where $k$ and $n$ are varying on the patient PK-PD characteristics. If one compares (6) with (22) it can be recognized the resemblance in the power term and observe in fact a simplification of the model from (5) in terms of number of model parameters. From a structural point of view, there is no difference between the models, since both are semi-empirical models. The term $\frac{C_{e}(t)}{B I S(t)}$ denotes the concentration-to-effect ratio (CER) and its units are $[\mathrm{mg} / \mathrm{ml} / \%]$.

In order to verify the validity of the above assumptions, a simulation study has been performed. Values for the effect site concentration $C_{e}(t)$ have been given in the range from $0.01-20 \mathrm{mg} / \mathrm{ml}$, in a linear distribution of 2000 points. $B I S(t)$ has been calculated with parameters from [22] and formula (5). The purpose of $k$ is that of a scaling factor, hence a common value has been identified for patients \#3, $\# 6$ and \#9 from figure 3: 5.45 . The identified values for the parameter $n$ in the same patients are $n=3.88, n=2.72$ and $n=4.45$, respectively. For the values of the $\gamma$ parameter which delivers the Hill curves in figure 3 we observe that patients \#6 and \#9 have the the highest and the lowest values. We correlate this observation with the values identified for the $n$ parameter and hence we may conclude that the $n$ parameter holds a similar significance in (22) as that of the $\gamma$ parameter in (5).

The results of the identification with the new PD model from (22) for the three simulated patients are given in figure 5.

\section{CHALLENGES AND CONCLUSIONS}

It is obvious that i) closed loop control is necessary and ii) an averaged patient model will deliver sub-optimal results since they require a robust, conservative controller able to deal with the inter-patient variability. Moreover, it is also clear that the inter-patient variability may vary quite significantly and that one single controller (without online adaptation) will never suffice in practice.

A first problem is to find online adaptation algorithms which may adapt the model parameters $k$ and $n$ to the patient
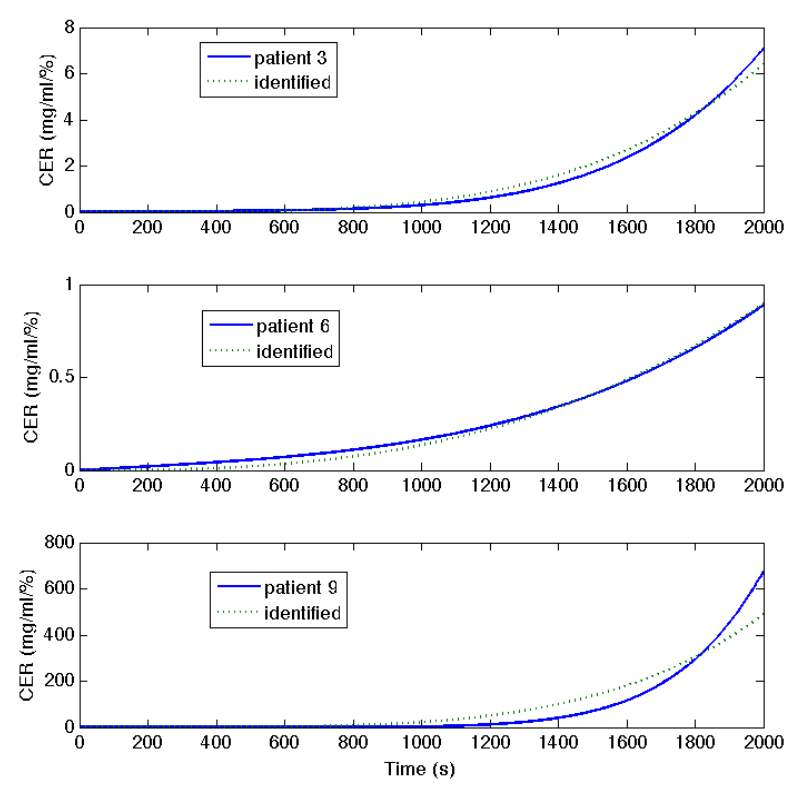

Fig. 5. Result of identification for a selection of three outlier patients.

characteristics. Identification from logarithmically sampled data has been proposed in [37] and represents a good framework for developing the online identification algorithm.

The next problem is to find a solution to integrate the model from (22) into a closed loop control system taking into account the requirement for a logarithmic sample time (i.e. in order to maintain linearity). Although it may look suprisingly, it has already been shown in various examples that a Riemann sampling rate (i.e. linear periodic) may be outperformed by a Lebesque sampling rate (i.e. eventtriggered) in several applications [38]. Since the Lebesque sampling rate is an event trigerred rate used successfully in practice in closed loop control (e.g. networked control, sensor networks), it may be revealing to look into the possibility of a logarithmic sampling rate.

This paper presented the available tools emerging from fractional calculus to model the nonlinear characteristics of the pharmacokinetic and pharmacodynamic patient models. Advantages and challenges have been discussed. Results suggest that the high degree of inter-patient variability and nonlinearity may be avoided, leading to linear control techniques instead of advanced, complex control techniques.

Further steps of this research line are dealing with the current limitations: i) online identification of logarithmically sampled data and ii) control of logarithmically sampled systems.

\section{ACKNOWLEDGMENT}

C. M. Ionescu acknowledges the Flanders Research Center (FWO) for its financial support. D. Copot acknowledges the support of the project FWO nr. FWOPR2014 002601. 


\section{REFERENCES}

[1] Bailey J. M. and Haddad W. M., "Drug dosing control in clinical pharmacology", IEEE Control Systems Magazine, vol. 25, no. 2, pp. 35-51, Apr. 2005

[2] Haddad W. M., Hayakawa T. and Bailey J. M. , "Nonlinear adaptive control for intensive care unit sedation and operating room hypnosis", American Control Conference, vol. 2, pp. 1808-1813, 2003.

[3] O'Hara D., Bogen D. and Noordergraaf A., "The use of computers for controlling the delivery of anesthesia", Anesthesiology, vol. 77, pp. 563-581, 1992

[4] Greenhow S. G., Linkens D. A. and Asbury A. J. , "Pilot study of an expert system adviser for controlling general anesthesia", British Journal of Anesthesia, vol. 71, pp. 359-365, 1993.

[5] Struys M, Vereecke H, Moerman A, Jensen EW, Verhaeghen D, De Neve N, Dumortier FJ, Mortier EP. "Ability of the bispectral index, autoregressive modeling with exogenous input-derived auditory evoked potentials and predicted propofol concentrations to measure patient responsiveness during anaesthesia", Anesthesiology, vol. 99, pp. 802-812, 2003.

[6] Absalom A.R., De Keyser R., Struys M.M.R.F., "Closed Loop Anesthesia: Are We Getting Close to Finding the Holy Grail?", Anesthesia and Analgesia, vol. 112, no. 3, pp. 364-381, 2011.

[7] Goutelle S., Maurin M., Rougier F., Barbaut X., Bourguignon L., Ducher M., Maire P. , "The Hill equation: a review of its capabilities in pharmacological modeling", Fundamental and Clinical Pharmacology, vol. 22, issue 6, pp. 633-648, 2008.

[8] Kress J., Pohlman A. and Hall J., "Sedation and analgesia in the intensive care unit", American Journal of Respiratory and Critical Care Medicine, vol. 166, pp. 1024-1028, 2002.

[9] Schnider T.W., Minto C.F., Gambus P.L. , Andresen C, Goodale DB , Youngs EJ, The influence of method of administration and covariates on the pharmacokinetics of Propofol in adult volunteers, Anesthesiology, 88: 11701182, 1998

[10] Schnider TW., Minto CF, Shafer SL, Gambus PL, Andresen C, Goodale DB, Youngs EJ, The influence of age on Propofol pharmacodynamics, Anesthesiology, 90:1502-16, 1999

[11] Macheas P., Iliadis, A. Modeling in Biopharmaceutics, Pharmacokinetics, and Phamacodynamics: Homogeneous and Heterogeneous Approaches, Springer Verlag, New York, 2006.

[12] Absalom AR, Mani V, De Smet T, Struys MM., "Pharmacokinetic models for propofoldefining and illuminating the devil in the detail", British Journal of Anaesthesiology, vol. 103, pp. 26-37, 2009.

[13] Liu N, Chazot T, Genty A, Landais A, Restoux A, McGee K, Laloe PA, Trillat B, Barvais L, Fischler M., "Closed-loop coadministration of propofol and remifentanil guided by bispectral index: a randomized multicenter study", Anesthesia and Analgesia, vol. 112, issue 3, pp. 546-557, 2011.

[14] Dumont G, Martinez A, Ansermino M., "Robust control of depth of anaesthesia, International Journal of Adaptive Control Signal Processing, vol. 23, pp. 435-454, 2009.

[15] Denai M., Linkens D. A., Asbury A. J., MacLeod A. D. and Gray W. M., Self-tuning PID control of atracurium-induced muscle relaxation in surgical patients, IEE Proc.-D, vol. 137, pp. 261-272, 1990.

[16] Mendonca T., Lago P. and Esteves S., "Hipocrates: a robust system for the control of neuromuscular blockade", Journal Clinical Monitoring and Computing, vol. 18, pp. 265-273, 2004.

[17] Melzack R., "Pain-an overview", Acta Anaesthesiologica Scandinavica, vol. 43, pp. 880-884, 1999.

[18] Minto C. F., White M., Morton N. and Kenny G. N., "Pharmacokinetics and pharmacodynamics of remifentanil. II Model application", Anesthesiology, vol. 86, pp. 24-33, 1997.

[19] Mortier E., Struys M., De Smet T., Versichelen L., Rolly G., Closedloop controlled administration of propofol using bispectral analysis, Anaesthesia, 53:749-754, 1998

[20] Jacques J.A., Compartmental analysis in biology and medicine, University of Michigan Press, 1985

[21] Berg J.M., ed., Biochemistry, 6th ed., New York City, NY: W.H. Freeman and Company, 2002.

[22] Ionescu C.M., De Keyser R., Torrico B. C., De Smet T., Struys M. and Normey-Rico J. E., "Robust Predictive Control Strategy Applied for Propofol Dosing using BIS as a Controlled Variable during Anesthesia", IEEE Transactions on Biomedical Engineering, vol. 55, pp. 2161-2170, 2008.
[23] Rinaki E., Valsami G., Macheras P., "The power law can describe the 'entire' drug release curve from HPMC-based matrix tablets: a hypothesis", Int J of Pharmaceutics, 255, 199-207, 2003

[24] Higuchi T., "Rate of release of medicaments from ointment bases containing drugs in suspension", J. Pharm Sci, 50, 874-875, 1961

[25] Kopelman R., "Fractal reaction kinetics", Science, 241, 1620-1626, 1988

[26] Tenreiro Machado J.A., Kiryakova V., Mainardi F., Recent history of fractional calculus, Communications in Nonlinear Science and Numerical Simulation, 16(3), 1140-1153, 2013

[27] Magin, R. (2006). Fractional Calculus in Bioengineering. Begell House Publishers.

[28] Macheras P, "A fractal approach to heterogeneous drug distribution: calcium pharmacokinetics", Pharmaceutical Research, vol. 13, pp. 663-670, 1996

[29] Dokoumetzidis A, Macheras P., "Fractional kinetics in drug absorption and disposition processes", Journal of Pharmacokinetics and Pharmacodynamics, vol. 36, pp. 165-178, 2009.

[30] Popovic JK, Atanackovic MT, Pilipovic AS, Rapaic MR, Pilipovic S, Atanackovic TM., "A new approach to the compartmental analysis in pharmacokinetics: fractional time evolution of diclofenac", Journal of Pharmacokinetics and Pharmacodynamics, vol 37, issue 2, pp. 135136, 2010.

[31] Dokoumetzidis A, Magin R., Macheras P. "A commentary on fractionalization of multi-compartmental models", Journal of Pharmacokinetics and Pharmacodynamics, vol. 37, issue 2, pp. 203-207, 2010.

[32] Copot D., Chevalier A, Ionescu C., De Keyser R, A two-compartment fractional derivative model for propofol diffusion in anesthesia, Conference on Systems and Control, Hyderabad, India, 28-31 August 2013, 264-269, 2013

[33] Ionescu C.M., Copot D., De Keyser R., Three compartmental model for propofol diffusion during general anesthesia, Discontinuity, Nonlinearity and Complexity, 2(4), 357-368, 2013

[34] Ionescu C., The human respiratory system: an analysis of the interplay between anatomy, structure, breathing and fractal dynamics, Springer, Series in BioEngineering 2013, Print ISBN 978-1-4471-5387-0, Online ISBN 978-1-4471-5388-7

[35] Tucker GT, Jackson PR, Storey GC, Holt DW, "Amiodarone disposition: polyexponential, power and gamma functions", European Journal of Clinical Pharmacology, vol. 26, pp. 655-656, 1984.

[36] Wise ME, "Negative power functions of time in pharmacokinetics and their implications", Journal of Phamacokinetics and Biopharmaceutics, vol. 13, pp. 309-346, 1985.

[37] De Keyser R., Ionescu C., Festila C., A one-step procedure for frequency response estimation based on switch-mode transfer function analyzer, Proc. IEEE-CDC Conf on Decision and Control joint with ECC-European Control Conf., December 12-15, Orlando, Florida, 1189-1194, 2011

[38] Goodwin G., Aguero J.C., Cea-Garrido M.E., Salgado M.E., Yuz J.I., "Sampling and sampled-data models", IEEE Control Systems Magazine, 33(5), 34-53, 2013 